\title{
Promoting Mobility and Reducing Length of Stay in Hospitalized General Medicine Patients: A Quality-Improvement Project
}

\author{
Erik H. Hoyer, MD ${ }^{1,2 \star}$, Michael Friedman, PT, MBA ${ }^{1,3}$, Annette Lavezza, OT ${ }^{3}$, Kathleen Wagner-Kosmakos, RN, MS ${ }^{4}$, \\ Robin Lewis-Cherry, RN4 , Judy L. Skolnik, RN, BSN4, Sherrie P. Byers, RN, MSN4, Levan Atanelov, MD', \\ Elizabeth Colantuoni, PhD5 , Daniel J. Brotman, MD², Dale M. Needham, FCPA, MD, PhD ${ }^{1,6}$
}

\begin{abstract}
${ }^{1}$ Department of Physical Medicine and Rehabilitation, Johns Hopkins University, Baltimore, Maryland; '2Division of General Internal Medicine, Johns Hopkins University, Baltimore, Maryland; ' ${ }^{3}$ Department of Physical Medicine and Rehabilitation, Johns Hopkins Hospital, Baltimore, Maryland; ${ }^{4}$ Department of Nursing, Johns Hopkins Hospital, Baltimore, Maryland; ${ }^{5}$ Department of Biostatistics, Johns Hopkins School of Public Health, Johns Hopkins University, Baltimore, Maryland; 'Division of Pulmonary and Critical Care Medicine, Johns Hopkins University, Baltimore, Maryland.
\end{abstract}

OBJECTIVE: To determine whether a multidisciplinary mobility promotion quality-improvement (QI) project would increase patient mobility and reduce hospital length of stay (LOS).

PATIENTS AND METHODS: Implemented using a structured QI model, the project took place between March 1, 2013 and March 1, 2014 on 2 general medicine units in a large academic medical center. There were 3352 patients admitted during the QI project period. The Johns Hopkins Highest Level of Mobility (JH-HLM) scale, an 8-point ordinal scale ranging from bed rest (score $=1$ ) to ambulating $\geq 250$ feet (score $=8$ ), was used to quantify mobility. Changes in $\mathrm{JH}$-HLM scores were compared for the first 4 months of the project (ramp-up phase) versus 4 months after project completion (post-QI phase) using generalized estimating equations. We compared the relative change in median LOS for the project months versus 12 months prior among the QI units, using multivariable linear regression analysis adjusting for 7 demographic and clinically relevant variables.

RESULTS: Comparing the ramp-up versus post-QI phases, patients reaching JH-HLM's ambulation status increased from $43 \%$ to $70 \%$ ( $P<0.001)$, and patients with improved $\mathrm{JH}-\mathrm{HLM}$ mobility scores between admission and discharge increased from $32 \%$ to $45 \%(P<0.001)$. For all patients, the QI project was associated with an adjusted median LOS reduction of 0.40 (95\% confidence interval [Cl]: -0.57 to $-0.21, P<0.001)$ days compared to 12 months prior. A subgroup of patients expected to have a longer LOS (expected LOS $>7$ days), were associated with a significantly greater adjusted median reduction in LOS of 1.11 (95\% Cl: -1.53 to $-0.65, P<0.001)$ days. Increased mobility was not associated with an increase in injurious falls compared to 12 months prior on the QI units $(P=0.73)$.

CONCLUSIONS AND RELEVANCE: Active prevention of a decline in physical function that commonly occurs during hospitalization may be achieved with a structured QI approach. In an adult medicine population, our QI project was associated with improved mobility, and this may have contributed to a reduction in LOS, particularly for more complex patients with longer expected hospital stay. Journal of Hospital Medicine 2016;11:341-347. (C) 2016 Society of Hospital Medicine
Annually, more than 35 million patients are hospitalized in the United States, with many experiencing hospital-acquired impairments in physical functioning during their in-patient stay. ${ }^{1-4}$ Such impairments include difficulties performing basic activities of daily living, such as rising from a chair, toileting, or ambulating. This functional decline may result in increased length of stay (LOS), nursing home placement, and decreased mobility and participation in community activities even years after hospitalization. ${ }^{1-3,5-7}$ Ameliorating this hospital-acquired functional impairment is important to improving patient outcomes and reducing healthcare utilization. Even the sickest hospitalized

*Address for correspondence and reprint requests: Erik H Hoyer, MD, 600 N Wolfe Street, Phipps 174, Baltimore, MD 21287; Telephone: 410-502-2438; Fax: 410-502-2419; E-mail: ehoyer1@jhmi.edu

Additional Supporting Information may be found in the online version of this article.

Received: March 10, 2015; Revised: December 5, 2015; Accepted: December 17, 2015

2016 Society of Hospital Medicine DOI 10.1002/jhm.2546

Published online in Wiley Online Library (Wileyonlinelibrary.com). patients (eg, those in the intensive care unit [ICU]), can safely and feasibly benefit from early mobilization. ${ }^{6,8-11}$ In the non-ICU setting there is also evidence that patient mobilization reduces LOS and hospital costs, while improving patient satisfaction and physical and psychological outcomes. ${ }^{12-16}$ These studies are, however, difficult to replicate as part of routine clinical care, because they often do not present the details of how early mobility was incorporated into daily practice, require additional hospital resources (eg, specially trained providers or additional staff), or are focused only on a select patient population.

The Johns Hopkins medical ICU started early rehabilitation quality-improvement (QI) work in 2007, which has demonstrated ongoing reductions in LOS and been transformative in terms of helping to foster a "culture of mobility" at our institution. Previous research suggests that ICU-based rehabilitation interventions are often not carried over to the ward setting, even in post-ICU patients. ${ }^{17}$ Moreover, trends for sicker patients being admitted in our general medicine units, ${ }^{18}$ growing reports of patients spending 
most of their time in bed,,$^{2,19,20}$ and healthcare policies emphasizing the importance of improving inpatient outcomes motivated the need for QI to improve patient mobility in this setting. Experience from the medical ICU-based early rehabilitation program helped drive multidisciplinary collaboration of stakeholders to develop this nurse-driven, mobility promotion QI project on 2 general medicine hospital units. The main goals of the project were to see whether a QI framework can be used in a general medicine setting to increase patient mobility and reduce LOS. ${ }^{21,22}$

\section{METHODS}

\section{Overview of Project}

Mobility, for this project, was defined as a patient getting out of bed (eg, sitting out of bed, toileting at bedside commode or bathroom, standing, and ambulating). We aimed to increase patient mobility using preexisting unit staffing ratios of clinicians and support staff. This project was reported in accordance with the SQUIRE (Standards for QUality Improvement Reporting Excellence) guidelines and used a structured QI model that had been used to successfully promote early mobility in the intensive care unit. $^{21,23-25}$ The planning phase of the QI project began in spring 2012, with initiation of the 12-month project on March 1, 2013. During the 12-month QI period, prospective collection of mobility status occurred for all patients, with no exclusions based on patient characteristics.

\section{Setting}

The QI project setting was 2, 24-bed, general medicine units at the Johns Hopkins Hospital, a large academic medical center located in Baltimore, Maryland.

\section{QI Process}

The primary goals of the QI project were to mobilize patients 3 times daily, quantify and document the mobility of the patients, set daily goals to increase mobility (eg, move up 1 step on the scale today), and standardize the description of patient mobility across all hospital staff. We used a structured QI model that that has been used to implement an early mobility program in a medical ICU at our institution ${ }^{21,22,24}$ (see Supporting Information, Appendix, in the online version of this article). At a programmatic level, we involved key stakeholders (nurses, physicians, rehabilitation therapists, administrators) in the QI project team, we identified local barriers to implementation through team meetings as well as a survey tool to identify perceived barriers, ${ }^{26}$ and we developed a scale (the Johns Hopkins Highest Level of Mobility [JH-HLM]) to document mobility. The JH-HLM is an 8-point ordinal scale that captures mobility milestones, where $1=$ only lying, $2=$ bed activities, $3=$ sit at edge of bed, $4=$ transfer to chair/commode, $5=$ standing for $\geq 1$ minute, $6=$ walking $10+$ steps,
$7=$ walking $25+$ feet, and $8=$ walking $250+$ feet (see Supporting Information, Appendix and Supporting Figure 1, in the online version of this article for additional information on the JH-HLM scale).

The 12-month QI project was characterized by several phases and milestones and involved a number of intervention components. During the first 4 months (ramp-up phase), nurses received education in the form of unit-based presentations, hands-on-training, and online education modules. On a 5-times weekly basis, nurses met with rehabilitation therapists for unit-based huddles to discuss baseline patient mobility, current patient mobility levels, barriers to mobilizing patients, and daily goals to progress mobility. Mobility levels were included on daily nursing report sheets to facilitate communication with subsequent shifts. Discussion of JH-HLM scores also occurred during daily unit-based care-coordination meetings of the nurses, physicians, and social-workers to address barriers to mobilizing patients, such as optimizing pain control, facilitating discharge location planning, and expediting physician consultation with physical and occupational therapy for appropriate patients. Audit and feedback from huddles and carecoordination rounds resulted in improved nurse attendance and engagement during these meetings. Nurses were expected to document patient mobility scores using the JH-HLM 3 times daily in the patient medical record. On the fourth month, reports on JHHLM scores and documentation compliance were available to nurse managers, champions, and unit staff. Via twice-monthly meetings with the units and quarterly meetings with hospital leadership and administration, problems arising during the QI intervention were evaluated and resolved on a timely basis. Seven months after project execution started, educational sessions were repeated to all staff, and feedback was provided based on the data collected, such as documentation compliance rates and patient mobility levels, and nurse champions presented the project during an American Nurses Credentialing Center magnet recognition program visit. Lastly, mobility scores and documentation compliance were continually assessed for 4 months after the project completion to determine sustainability of the intervention. Additional details of the QI project implementation are provided in the Supporting Information, Appendix, in the online version of this article.

\section{Data Sources and Covariates for Project Evaluation}

The Sunrise Clinical Manager system (Allscripts Healthcare Solutions Inc., Chicago, IL) was used to document and extract nursing-documented JH-HLM scores. The Johns Hopkins Hospital Datamart financial database, used for mandatory reporting to the State of Maryland, provided data on LOS, age, sex, race (white, black, other), payer (Medicare, Medicaid, other), primary admission diagnosis, and comorbidity 


\begin{tabular}{lcc} 
TABLE 1. Patient Characteristics on the QI Units* \\
\hline $\begin{array}{l}\text { Comparison Period, } \\
\text { March 2012-March 2013, }\end{array}$ & $\begin{array}{c}\text { QI Period, March } \\
\text { 2013-March 2014, } \\
\text { N }=3,302\end{array}$ & N $=3,352$ \\
\hline Characteristics & $53.3(17.8)$ & $54.4(18.3)$ \\
Age, $y$ & $1467(44 \%)$ & $1569(47 \%)$ \\
Male & & \\
Race & $1883(57 \%)$ & $1809(54 \%)$ \\
African American & $1269(38 \%)$ & $1348(40 \%)$ \\
Caucasian & $150(5 \%)$ & $195(6 \%)$ \\
Other & & \\
Payer & $1310(40 \%)$ & $1470(44 \%)$ \\
Medicare & $1015(31 \%)$ & $925(28 \%)$ \\
Medicaid & $977(30 \%)$ & $957(29 \%)$ \\
Other & & \\
Admission diagnostic category & $579(18 \%)$ & $629(19 \%)$ \\
Infectious disease & $519(16 \%)$ & $559(17 \%)$ \\
Pulmonary & $535(16 \%)$ & $494(15 \%)$ \\
Gastrointestinal & $410(12 \%)$ & $405(12 \%)$ \\
Cardiovascular & $199(6 \%)$ & $195(6 \%)$ \\
Hematologic & $220(7 \%)$ & $205(6 \%)$ \\
Renal & $840(25 \%)$ & $865(26 \%)$ \\
Other & $5.5(3.3)$ & $5.3(3.2)$ \\
UHC expected length of stay, d & $3.3(1.7)$ & $3.5(1.8)$ \\
AHRQ comorbidity index & & \\
& &
\end{tabular}

NOTE: Abbreviations: AHRQ, Agency for Healthcare Research and Quality; Ql, quality improvement; UHC, University Health Consortium. ${ }^{*}$ Continuous variables are presented as mean (standard deviation), and dichotomous variables are presented as $n(\%)$

index using Agency for Healthcare Research and Quality (AHRQ) methodology. ${ }^{27}$ Expected LOS was calculated using the risk adjustment method developed by the University Health System Consortium (UHC). ${ }^{28}$ This calculation uses a combination of the Diagnostic-Related Group grouper and the Sachs Complication Profiler ${ }^{29}$ in conjunction with data on specific patient characteristics (age, sex, urgency of admission, payer category) to construct riskadjustment regression models that assign expected values for LOS, and is not based on actual LOS. ${ }^{28}$ The databases were linked at the patient level using the patient's medical record and unique admission record number.

\section{Outcome Measures}

Two functional outcome measures were based on daily JH-HLM scores, which frequently occurred several times on each patient-day: (1) the maximum daily JH-HLM scores for each patient-day during hospitalization, and (2) the intrapatient change in JH-HLM scores between the maximum JH-HLM score within 24 hours of hospital admission and 24 hours before discharge for all patients who were on the unit $>48$ hours. We also compared the mean LOS during the 12-month QI project versus the 12-months prior so we could more accurately address seasonal differences. $^{30-35}$ Lastly, because the perception of increased falls was an important barrier to address in the QI process, we compared the rate of injurious falls between the QI period and 12-months prior.

\section{Statistical Analysis}

To evaluate changes in the percent of ambulatory patients (JH-HLM $\geq 6$ ), we compared the initial 4 months of the QI project (ramp-up phase) with the same 4-month period occurring immediately after project completion (post-QI phase) using generalized estimating equations to account for clustering at the patient-level. This test was also used to evaluate changes in documentation compliance rates between the 2 phases, with compliance defined as at least 1 instance of JH-HLM documentation per day, excluding the day of admission and discharge. To evaluate if improved JH-HLM results were driven by improved documentation compliance rates over time, we performed a sensitivity analysis by imputing a JH-HLM score of 6 (ambulate $10+$ steps) for any missing daily maximum JH-HLM scores.

To assess unadjusted changes in LOS during the 12month QI project versus the same period 1 year earlier, we compared mean and median LOS using a $t$ test and Wilcoxon rank sum test, respectively. We used a multivariable linear regression model to estimate the change (expressed in days) in adjusted median LOS comparing the project months (March 2013-March 2014) with 12 months prior (March 2012-March 2013). The model adjusted for age, gender, race, payer, admission diagnostic category, UHC expected LOS, and AHRQ comorbidity index. We confirmed a lack of multicollinearity in the multivariable regression model using variance inflation factors. We evaluated residual versus predicted value plots and residual versus fitted value plots with a locally weighted scatterplot smoothing line to confirm model fit. $P$ values are reported from the test of the null hypothesis that the change in adjusted median LOS is the same comparing the QI project months versus 12 months prior. Separate models estimated and tested the change in adjusted median LOS by tertiles of expected LOS $(<4,4-7$, and $>7$ days). Lastly, we compared the rate of injurious falls (the number of injurious falls by total patient-days) between the QI period and 12 months prior using an exact Poisson method. ${ }^{36}$ Statistical significance was defined as a 2 -sided $P<0.05$. Statistical analyses were conducted using R (version 3.1.0; The R Foundation for Statistical Computing, Vienna, Austria; http://www.r-project. org). This study was approved, with waiver of consent, by the Johns Hopkins Institutional Review Board as a research project.

\section{RESULTS}

During the QI project period, 3352 patients were admitted to the 2 general medicine units. Twelve $(0.4 \%)$ patients expired on the units, but their data were retained in the analysis. Mean (standard deviation [SD]) age of the patients was 54.4 (18.3) years, with $47 \%$ male, and $54 \%$ African American. A total of 1896 of 6654 (28\%) patients on the QI units were 
TABLE 2. Change in Mobility Scores During the 12-Month QI Project and the First 4 Months Thereafter

\begin{tabular}{|c|c|c|c|}
\hline JH-HLM Category & $\begin{array}{l}\text { Ramp-up Phase, March 1, } 2013 \text { - June 30, } \\
\qquad 2013, n=4,649\end{array}$ & $\begin{array}{l}\text { Late-QI Phase, November 1, 2013-February } \\
\qquad 28,2013, n=4,515\end{array}$ & $\begin{array}{l}\text { Post-QI Phase, March 1, 2014- June 30, } \\
\qquad 2014, n=4,298\end{array}$ \\
\hline Walk (JH-HLM $=6,7$, or 8$)$ & $1,994(43)$ & $3,430(76)$ & $2,986(70)$ \\
\hline Stand/chair (JH-HLM = 4 or 5) & $1,772(38)$ & $488(10)$ & $511(12)$ \\
\hline $\operatorname{Bed}($ JH-HLM $=1,2$, or 3$)$ & $883(19)$ & $597(13)$ & $801(19)$ \\
\hline $\begin{array}{l}\text { Change in Mobility (Admission } \\
\text { Versus Discharge) }\end{array}$ & $\begin{array}{l}\text { Ramp-up Phase, March 1, } \\
\text { 2013-June 30, 2013, n = } 968\end{array}$ & $\begin{array}{l}\text { Late-QI Phase, November } 1 \text {, } \\
\text { 2013-February 28, 2013, } \mathrm{n}=893\end{array}$ & $\begin{array}{l}\text { Post-QI Phase, March 1, } \\
\text { 2014- June 30, 2014, n = } 834\end{array}$ \\
\hline Improved & $305(32)$ & $392(44)$ & $379(45)$ \\
\hline No change & $512(53)$ & $428(48)$ & $386(46)$ \\
\hline Declined & $151(16)$ & $73(8)$ & $69(8)$ \\
\hline
\end{tabular}

NOTE: Change in patient mobility during the 12-month QI project and the 4 months after completion of the project, using the Johns Hopkins Highest Level of Mobility (JH-HLM) scale. Values are presented as n (\%). For all analyses, the maximum daily JH-HLM score was used for each patient-day of data. The top section refers to the percentage of patient-days with mobility scores in each of the JH-HLM categories (walk, stand/chair, bed). The bottom section refers to the percentage of patients in each category (improved, no change, declined) based on the difference in their discharge JH-HLM scores compared to their admission scores for patients who were on the unit > 48 hours. Abbreviations: Ql, quality improvement.

TABLE 3. Comparison of the Absolute Change in Adjusted Median LOS for the Project Months Versus 12-Months Prior* $^{*}$

\begin{tabular}{|c|c|c|c|c|}
\hline & \multicolumn{2}{|c|}{ Adjusted Median LOS, d } & \multirow[b]{2}{*}{ Absolute Change in Adjusted Median LOS (95\% Cl), d } & \multirow[b]{2}{*}{$P$ Value $^{\dagger}$} \\
\hline & 12 Months Prior & QI Project Months & & \\
\hline All patients & 6.01 & 5.61 & $-0.40(-0.57$ to -0.21$), N=4,411$ & $<0.001$ \\
\hline \multicolumn{5}{|l|}{ Subgroups by ELOS ${ }^{\ddagger}$} \\
\hline ELOS $<4$ days & 4.68 & 4.77 & $0.09(-0.13$ to 0.32$), \mathrm{N}=1,357$ & 0.42 \\
\hline ELOS 4-7 days & 5.68 & 5.38 & $-0.30(-0.57$ to -0.01$), N=1,509$ & 0.04 \\
\hline ELOS $>7$ days & 8.07 & 6.96 & $-1.11(-1.53$ to -0.65$), \mathrm{N}=1,545$ & $<0.001$ \\
\hline
\end{tabular}

NOTE: Abbreviations: AHRQ, Agency for Healthcare Research and Quality; Cl, confidence interval; ELOS, expected length of stay; LOS, length of stay, QI, quality improvement; UHC, University Health Consortium. *Absolute change (expressed in days) in adjusted median LOS compared project months (March 2013-March 2014) with 12 months prior (March 2012-March 2013) and were calculated using a linear regression analysis for the logarithm of LOS. Patients with an LOS $>48$ hours were included in the analyses. Analyses were adjusted for age, sex, race, payer, admission diagnostic category, UHC expected LOS, and AHRQ comorbidity index. ${ }^{\dagger} P$ values are reported from the test of the null hypothesis that the change in adjusted median LOS is the same comparing the QI project months versus 12 months prior. ${ }^{~}$ Separate models estimated and tested the change in adjusted median LOS by tertiles of UHC expected LOS $(<4,4-7$, and $>7$ days).

$\geq 65$ years old. Patient characteristics were similar during the QI period versus 12 months prior (Table 1).

During the 12-month QI project, there were a total of 13,815 patient-days of documented mobility data and the median (interquartile range [IQR]) number of days of documentation for each hospital admission was $3(2-5)$ days. Compliance with daily documentation of JH-HLM was $85.0 \%$ over the entire 12 -month QI project. Documentation compliance started at $83 \%$ during the ramp-up phase and increased to $89 \%$ during the last 4 months of the project (late-QI phase, $P<0.001$ ).

Comparing the ramp-up phase versus post-QI phase, the percentage of patient-days in which patients ambulated (JH-HLM $\geq 6$ ) increased from $43 \%$ to $70 \%(P<0.001)$, and the percentage of patients who experienced an improvement in their mobility scores between admission and discharge increased from $32 \%$ to $45 \%(P<0.001)$, as shown in Table 2. In the sensitivity analysis imputing missing daily JH-HLM scores and comparing the ramp-up versus post-QI phases, the results were similar to the primary analysis; the percent of patient-days where patients ambulated increased from $60 \%$ to $78 \%(P<$ $0.001)$, and the percent of patients who experienced an improvement in their mobility scores increased from $26 \%$ to $48 \%(P<0.001)$.

LOS during the 12-month QI project versus the 12 -months immediately prior was shorter (Table 3 ), with an unadjusted median (IQR) LOS of $3(2-6)$ versus $4(2-7)$ days $(P<0.001)$ and an unadjusted mean (SD) LOS of $5.1(5.6)$ versus $6.0(7.6)(P<0.001)$.

Table 3 displays the change in adjusted median LOS for the project months versus the 12 months prior among the QI units. We found that for all patients, there was an overall reduction in adjusted median LOS of 0.40 (95\% confidence interval [CI]: -0.57 to $-0.21, P<0.001)$ days. When we divided patients into tertiles based on their UHC expected LOS (ELOS), we observed that patients with longer ELOS had greater reductions in adjusted median LOS. Patients on the QI units with ELOS $<4$ days (lowest tertile) did not show a significant reduction in adjusted median LOS (0.09 days, 95\% CI: -0.13 to $0.32, P=0.42$ ); however, patients with UHC ELOS 4 to 7 days (middle tertile) and ELOS $>7$ days (highest tertile) had a significant reduction in adjusted median 
LOS by 0.30 (95\% CI: -0.57 to $-0.01, P=0.04)$ and 1.11 (95\% CI: -1.53 to $-0.65, P<0.001)$ days during the QI project versus 12 months prior, respectively.

Lastly, we found that there was no difference in the rate of injurious falls on the QI units during QI period compared to 12 months prior (QI: 0.34 per 1000 patient-days versus 12 months prior: 0.48 per 1000 patient-days, $P=0.73$ ).

\section{DISCUSSION}

We conducted a nurse-driven, multidisciplinary mobility promotion QI project on 2 general medicine units at a large teaching hospital. The 12-month QI project, conducted between March 1, 2013 and February 28, 2014, was associated with patients ambulating more frequently, with improved mobility status between hospital admission and discharge. These improvements in mobility were not associated with increased rates of injurious falls, and were sustained for at least 4 months after project completion. The QI project was associated with overall significant reduction in LOS for more complex patients with longer expected LOS (4 days or longer). Hence, such QI efforts may be important for maintaining or improving patients' functional status during hospitalization in a safe and cost-effective manner.

Our findings are consistent with previous studies showing that mobility promotion in the acute hospital setting is feasible, can reduce length of stay, and can be applied to a diverse population including vulnerable medical patients with multiple comorbidities and the elderly. ${ }^{12,16,37-42}$ These studies provide valuable evidence of the benefits of mobility promotion; however, it is difficult to translate these prior results into routine clinical practice because they used specially trained staff to mobilize patients, focused on a select patient population, or did not specify how the mobility intervention was delivered within daily clinical workflows. Research in the medical ICU at our institution has previously described the use of a structured QI model to successfully implement an early rehabilitation program. ${ }^{22,24}$ Here, we successfully adapted the same QI framework to a general medicine setting. Hence, our study contributes to the literature with respect to (1) use of a structured QI framework to develop a successful patient mobility program in a general medicine patient population, and (2) sharing best practices from 1 clinical setting, such as the ICU, as a source of learning and knowledge translation for other care settings, with the addition of novel tools, such as the JH-HLM scale.

There may have been several factors that contributed to shorter stays in the hospital we observed during the QI project. First, we increased the number of ambulatory patient-days, which may have helped prevent physiological complications of bed rest, such as muscle weakness, atelectasis, insulin resistance, vascu- lar dysfunction, contractures, and pressure ulcers. ${ }^{43}$ As such, mobility promotion has been associated with reduced rates of other hospital-acquired complications, such as deep venous thrombosis, pneumonia, and delirium. ${ }^{44-46}$ In our study, we saw the greatest LOS reduction in more complex patients who were expected to spend a longer time in the hospital and are at greater risk of developing complications from bed rest. Second, our early mobility project may have had a direct impact on care-coordination processes as reported in prior studies. ${ }^{47-49}$ An important component of our intervention was incorporating functional status into multidisciplinary discussions, either through nurse-to-therapist huddles or carecoordination rounds between nurses, therapists, physicians, social workers, and case managers. During care-coordination rounds, JH-HLM scores were reported to expedite appropriate physical and occupational therapy consultations and assist in determining appropriate discharge location. During the QI project, we transitioned from a unit-based daily huddle between nursing and rehabilitation therapists to a system where mobility status was discussed primarily during care coordination rounds 5 times per week. We saw that mobility scores were maintained after QI project completion, suggesting that reporting on patient function in a multidisciplinary setting is a potentially sustainable mechanism to improve carecoordination processes that are affected by functional status.

Our study has several potential limitations. First, this is a single-site study in 2 general medicine units of a large academic hospital. Further research is needed to determine if this structured QI intervention and its benefits can be generalized to different settings and different patient populations. Second, because the documentation was initially an optional element in the electronic medical record system, we observed higher rates of missing documentation during the first 4 months of the project versus the comparison period at 4 months after project completion. However, a sensitivity analysis conducted of these missing data demonstrated similar results to our primary analysis. Third, our nonrandomized pre-post study design does not allow us to conclude a direct cause-and-effect relationship between our intervention and increased mobility and reduced LOS. Although patient characteristics were similar between the 2 periods and adjusted for in our multivariable regression analysis, we cannot rule out the possibility of secular trends in LOS on the project units and that broader QI efforts at our institution also contributed to reduction in LOS. Fourth, we do not have data on 30-day readmissions and discharge location. Future studies should explore the impact of hospital-based mobility interventions on these outcomes. ${ }^{50}$ Fifth, although nurses consistently documented the highest level of mobility on a daily basis, these data did not capture other 
potentially important information about patient mobility such as the daily frequency that patients were mobilized, the length of time a patient was engaged in a mobility event (ie, number of hours sitting in a chair), or the mobility that occurred during physical therapy or occupational therapy sessions. Hence, although we used JH-HLM as a marker of improved mobility during our QI project it is likely that our data cannot fully describe the total mobility and activity that patients experienced during hospitalization. Lastly, although the front-line staff and QI team found the JH-HLM scale to be a useful tool to measure and advance patient mobility, further studies are needed to evaluate the reliability and validity of this scale.

\section{CONCLUSION}

A structured QI process can improve patient mobility and may contribute to reduction in LOS, particularly for more complex patients in this setting. Active prevention of decline in physical function that commonly occurs during hospitalization may prove valuable for improving patient outcomes and reducing healthcare resource utilization.

\section{Disclosures}

The authors certify that no party having a direct interest in the results of the research supporting this article has or will confer a benefit on us or on any organization with which we are associated. The authors report no conflicts of interest.

\section{References}

1. Covinsky KE, Palmer RM, Fortinsky RH, et al. Loss of independence in activities of daily living in older adults hospitalized with medical illnesses: increased vulnerability with age. J Am Geriatr Soc. 2003; 51(4):451-458.

2. Brown CJ, Friedkin RJ, Inouye SK. Prevalence and outcomes of low mobility in hospitalized older patients. J Am Geriatr Soc. 2004;52(8): $1263-1270$.

3. Brown CJ, Roth DL, Allman RM, Sawyer P, Ritchie CS, Roseman JM. Trajectories of life-space mobility after hospitalization. Ann Intern Med. 2009;150(6):372-378.

4. Covinsky KE, Pierluissi E, Johnston CB. Hospitalization-associated disability: "She was probably able to ambulate, but I'm not sure". JAMA. 2011;306(16):1782-1793.

5. Blair SN, Kohl HW III, Paffenbarger RS Jr, Clark DG, Cooper KH, Gibbons LW. Physical fitness and all-cause mortality. A prospective study of healthy men and women. JAMA. 1989;262(17):2395-2401.

6. Needham DM. Mobilizing patients in the intensive care unit: improving neuromuscular weakness and physical function. JAMA. 2008; 300(14):1685-1690.

7. Brown CJ, Flood KL. Mobility limitation in the older patient: a clinical review. JAMA. 2013;310(11):1168-1177.

8. Schweickert WD, Pohlman MC, Pohlman AS, et al. Early physical and occupational therapy in mechanically ventilated, critically ill patients: a randomised controlled trial. Lancet. 2009;373(9678):1874-1882.

9. Needham DM, Truong AD, Fan E. Technology to enhance physical rehabilitation of critically ill patients. Crit Care Med. 2009;37(10 suppl):S436-S441.

10. Morris PE, Griffin L, Berry M, et al. Receiving early mobility during an intensive care unit admission is a predictor of improved outcomes in acute respiratory failure. Am J Med Sci. 2011;341(5):373-377.

11. Stiller K. Physiotherapy in intensive care: an updated systematic review. Chest. 2013;144(3):825-847.

12. de Morton NA, Keating JL, Jeffs K. Exercise for acutely hospitalised older medical patients. Cochrane Database Syst Rev. 2007;(1): CD005955.

13. Peiris CL, Taylor NF, Shields N. Extra physical therapy reduces patient length of stay and improves functional outcomes and quality of life in people with acute or subacute conditions: a systematic review. Arch Phys Med Rehabil. 2011;92(9):1490-1500.
14. Pashikanti L, Von Ah D. Impact of early mobilization protocol on the medical-surgical inpatient population: an integrated review of literature. Clin Nurse Spec. 2012;26(2):87-94.

15. Kalisch BJ, Lee S, Dabney BW. Outcomes of inpatient mobilization: a literature review. J Clin Nurs. 2014;23(11-12):1486-1501.

16. Stolbrink M, McGowan L, Saman H, et al. The early mobility bundle: a simple enhancement of therapy which may reduce incidence of hospital-acquired pneumonia and length of hospital stay. J Hosp Infect. 2014;88(1):34-39.

17. Hopkins RO, Miller RR III, Rodriguez L, Spuhler V, Thomsen GE. Physical therapy on the wards after early physical activity and mobility in the intensive care unit. Phys Ther. 2012;92(12):1518-1523.

18. Mendez CM, Harrington DW, Christenson P, Spellberg B. Impact of hospital variables on case mix index as a marker of disease severity. Popul Health Manag. 2014;17(1):28-34.

19. Callen BL, Mahoney JE, Grieves CB, Wells TJ, Enloe M. Frequency of hallway ambulation by hospitalized older adults on medical units of an academic hospital. Geriatr Nurs. 2004;25(4):212-217.

20. Kuys SS, Dolecka UE, Guard A. Activity level of hospital medical inpatients: an observational study. Arch Gerontol Geriatr. 2012; 55(2):417-421.

21. Pronovost PJ, Berenholtz SM, Needham DM. Translating evidence into practice: a model for large scale knowledge translation. BMJ. 2008;337:a1714.

22. Needham DM, Korupolu R, Zanni JM, et al. Early physical medicine and rehabilitation for patients with acute respiratory failure: a quality improvement project. Arch Phys Med Rehabil. 2010;91(4):536-542.

23. Davidoff F, Batalden P, Stevens D, Ogrinc G, Mooney SE; SQUIRE development group. Publication guidelines for quality improvement studies in health care: evolution of the SQUIRE project. BMJ. 2009; 338:a3152.

24. Needham DM, Korupolu R. Rehabilitation quality improvement in an intensive care unit setting: implementation of a quality improvement model. Top Stroke Rehabil. 2010;17(4):271-281.

25. Engel HJ, Needham DM, Morris PE, Gropper MA. ICU early mobilization: from recommendation to implementation at three medical centers. Crit Care Med. 2013;41(9 suppl 1):S69-S80.

26. Hoyer EH, Brotman DJ, Chan K, Needham DM. Barriers to early mobility of hospitalized general medicine patients: survey development and results. Am J Phys Med Rehabil. 2015;94(4):304-312.

27. Elixhauser A, Steiner C, Harris DR, Coffey RM. Comorbidity measures for use with administrative data. Med Care. 1998;36(1):8-27.

28. UHC Clinical Information Management Risk Adjustment of the UHC Clinical Data Base. Chicago, IL: University HealthSystem Consortium; 1998.

29. Sachs Complications Profiler, Version 1.0, User's Guide. Evanston, IL: Sachs Group; 1995

30. Jencks SF, Williams DK, Kay TL. Assessing hospital-associated deaths from discharge data. The role of length of stay and comorbidities. JAMA. 1988;260(15):2240-2246.

31. Martinez-Selles M, Garcia Robles JA, Prieto L, et al. Annual rates of admission and seasonal variations in hospitalizations for heart failure. Eur J Heart Fail. 2002;4(6):779-786.

32. Kinnunen T, Saynajakangs O, Tuuponen T, Keistinen T. Regional and seasonal variation in the length of hospital stay for chronic obstructive pulmonary disease in Finland. Int J Circumpolar Health. 2002;61(2): $131-135$.

33. Guru V, Anderson GM, Fremes SE, O’Connor GT, Grover FL, Tu JV; Canadian CABG Surgery Quality Indicator Consensus Panel. The identification and development of canadian coronary artery bypass graft surgery quality indicators. J Thorac Cardiovasc Surg. 2005; 130(5):1257.

34. Svendsen ML, Ehlers LH, Andersen G, Johnsen SP. Quality of care and length of hospital stay among patients with stroke. Med Care. 2009;47(5):575-582.

35. Peterson MC. A systematic review of outcomes and quality measures in adult patients cared for by hospitalists vs nonhospitalists. Mayo Clin Proc. 2009;84(3):248-254.

36. Fay MP. Confidence intervals that match Fisher's exact or Blaker's exact tests. Biostatistics. 2010;11(2):373-374.

37. Inouye SK, Bogardus ST Jr, Charpentier PA, et al. A multicomponent intervention to prevent delirium in hospitalized older patients. $N$ Engl J Med. 1999;340(9):669-676.

38. Mundy LM, Leet TL, Darst K, Schnitzler MA, Dunagan WC. Early mobilization of patients hospitalized with community-acquired pneumonia. Chest. 2003;124(3):883-889.

39. Chang P, Lai Y, Shun S, et al. Effects of a walking intervention on fatigue-related experiences of hospitalized acute myelogenous leukemia patients undergoing chemotherapy: a randomized controlled trial. J Pain Symptom Manage. 2008;35(5):524-534.

40. Fisher SR, Kuo YF, Graham JE, Ottenbacher KJ, Ostir GV. Early ambulation and length of stay in older adults hospitalized for acute illness. Arch Intern Med. 2010;170(21):1942-1943.

41. Padula CA, Hughes C, Baumhover L. Impact of a nurse-driven mobility protocol on functional decline in hospitalized older adults. J Nurs Care Qual. 2009;24(4):325-331. 
42. Mudge AM, Giebel AJ, Cutler AJ. Exercising body and mind: an integrated approach to functional independence in hospitalized older people. I Am Geriatr Soc. 2008;56(4):630-635.

43. Brower RG. Consequences of bed rest. Crit Care Med. 2009;37(10 suppl):S422-S428.

44. Kamel HK, Iqbal MA, Mogallapu R, Maas D, Hoffmann RG. Time to ambulation after hip fracture surgery: relation to hospitalization outcomes. J Gerontol A Biol Sci Med Sci. 2003;58(11):M1042M1045.

45. Chandrasekaran S, Ariaretnam SK, Tsung J, Dickison D. Early mobilization after total knee replacement reduces the incidence of deep venous thrombosis. ANZ J Surg. 2009;79(7-8):526-529.

46. Kurabe S, Ozawa T, Watanabe T, Aiba T. Efficacy and safety of postoperative early mobilization for chronic subdural hematoma in elderly patients. Acta Neurochir (Wien). 2010;152(7): 1171-1174.

47. Gittell JH, Fairfield KM, Bierbaum B, et al. Impact of relational coordination on quality of care, postoperative pain and functioning, and length of stay: a nine-hospital study of surgical patients. Med Care. 2000;38(8):807-819.

48. Care coordination cuts admissions, ED visits, LOS. Hosp Case Manag. 2013;21(5):67-68.

49. White SM, Hill A. A heart failure initiative to reduce the length of stay and readmission rates. Prof Case Manag. 2014;19(6):276-284.

50. Hoyer EH, Needham DM, Atanelov L, Knox B, Friedman M Brotman DJ. Association of impaired functional status at hospital discharge and subsequent rehospitalization. J Hosp Med. 2014;9(5): 277-282. 